\title{
The Beginning of the Parliamentary in Albania (1920-1924)
}

\author{
Prof.Assoc. Nertila Haxhia Ljarja \\ University of Shkodra "Luigj Gurakuqi" \\ Department of History
}

\section{Doi:10.5901/jesr.2014.v4n4p449}

\begin{abstract}
The years that followed post World War 1, proved to be a difficult time for the renovation of the Albanian population. A few radicalized Albanian patriots, with varying views on politics went as far as to hold meetings in the Congress with the common cause of improving the state of the country. These delegates were chosen from all different parts of the country and served as representatives of those parts. They were representing the country as well as its sovereignty. Of all these organizations, one in particular stood out. The Congress of Lushnja (28-31 January 1920) was the one to put best practice methods into place with regards to rebuilding Albania. As of this moment, the process of institutional and legislative europeanization in the country begins.
\end{abstract}

Keywords: government, parliament, election campaign.

\section{Le Origini del Parlamentarismo in Albania (1920-1924)}

In un momento critico per il futuro del paese, soprattutto dopo l'accordo Tittoni - Veniselos è stata necessaria una politica comune per proteggere i diritti e l'indipendenza degli albanesi. Da questo l'accordo, in congiunzione col confine meridionale, e dalle rivendicazioni italiane a rimanere in Vlora, per gli albanesi divenne chiaro che l'Italia non era nella loro difesa senza interesse, ma già si è visto, cosi come i greci e serbi hanno sostenuto di avere la loro quota in Albania, distruggendo le speranze albanesi per una Albania unita. ${ }^{1}$

\section{Congresso di Lushnja}

Tale situazione portò a una necessità di organizzare un congresso di patrioti albanesi a Lushnja, un'area al di fuori del controllo italiano, che si riunì il 28-31 gennaio 1920. Questo congresso formerà un governo centrale che rappresentasse tutti gli albanesi. La si sono riuniti 50 delegati da tutte le terre albanesi, tranne Kosovo e Çameria. Aqif Pashë Biçakçiu (Elbasani) ha diretto i lavori, una persona con sufficiente autorità. II Congresso ha preso diverse misure: ha sciolto il Governo Provvisorio di Durazzo definendolo responsabile per l'anarchia nel paese, completamente opposto da lei politicamente, è stata inviata una risoluzione alla Conferenza di Pace di Parigi, dove si protestava contro i piani di divisione d'Albania ed è stato nominato una delegazione di tre persone a rappresentare l'Albania lì; i membri del Congresso di Lushnja hanno chiesto al Senato degli Stati Uniti di intervenire a vantaggio della questione albanese, il Congresso ha nominato un Consiglio Supremo (con le funzioni di capo dello stato) di quattro persone, rappresentanti di credenze religiose mussulmane, bektashi, cattolici e ortodossi (Aqif Pashë Elbasani, Abdi Toptani, Mons. Luigi Bumçi, Dott.Mihal Turtulli)2 ${ }^{2}$, che avrebbero avuto il potere di un re o presidente e avrebbe funzionato fino all'arrivo di un re; è stato eletto un governo con sette membri sotto il primo ministro Sulejman Bej Delvina, Ministro degli Interni Ahmet Zogu e il Ministro dell'Istruzione Sotir Peci . II Congresso ha anche eletto un Consiglio Nazionale detto il Senato ${ }^{3}$, con le funzioni

\footnotetext{
${ }_{1}^{1}$ Romeo Gurakuqi, Shqipëria dhe çështja shqiptare pas Luftës së Parë Boterore. Botimet “Camaj-Pipa”, Shkodër, 2007, pg.115-116.

2 "Njëkohësisht këto katër anëtar duke përfaqësuar Elbasanin, Tiranën, Shkodrën dhe Korçën, ruajtën raportet krahinore të përfaqësimit. Në një aspekt tjetër, Aqif Elbasani përfaqësonte shtresën e pronarëve të tokave të lidhur me qeverinë e Vlorës dhe qarqet nacionaliste, Abdi Toptani traditën dhe familjet e mëdha të Tiranës, Luigj Bumçi, klerik katolik, kishte qenë një diplomat $i$ aftë në negociatat e Parisit dhe Dr.Turtulli përfaqësonte edhe emigracionin shqiptar në SHBA." Afrim Krasniqi, Sistemet politike në Shqipëri 1912-2008. Shtëpia Botuese UFO Press, Tiranë, mars 2009, pg.71.

3 "Një tjetër organ i lartë i shtetit shqiptar, që u vendos të krijohej në Kongresin e Lushnjës, ishte Senati. Në fakt natyra e këtij organi nuk pajtohej me emrin e tij. Në këtë rast, nuk kishin të bënin me një senat, si dhomë e dytë, që ndeshet zakonisht në parlamentet, por me një institucion që kryente funksionet e organit përfaqësues, të parlamentit të vendit. Ky karakter i senatit bëhej $i$ qartë si nga formulimi $i$ vendimeve të Lushnjës mbi pozitën e tij juridike, ashtu edhe nga vetë veprimtaria e më-vonshme e këtij organi, që nisi të funksiononte,
} 
del parlamento, con 37 membri in rappresentanza di dieci prefetture. La capitale albanese divenne Tirana, dove furono trasferiti gli organismi governativi.

II Congresso di Lushnja è stato un atto molto importante per determinare l'indipendenza dell'Albania senza mandato e protettorato straniero, per la determinazione del sistema di governo democratico e dei principi costituzionali del nuovo Stato. L'Albania continuerà ad essere una monarchia costituzionale, in cui il Consiglio Supremo di Stato esercita le funzioni del regno, dunque la monarchia sarà rappresentata non da una sola persona, ma da un organismo collegiale senza presidente. II completamento dei posti vacanti sarà effettuato dal Consiglio Nazionale, in particolare nei casi in cui la composizione del Consiglio Supremo sarebbe rimasta con meno di tre membri. Le sue decisioni avevano un carattere evidente democratico e si basavano su modelli occidentali governativi, fenomeno questo ancora sconosciuto negli stati emersi dall'Impero Ottomano. ${ }^{4}$ Questo organismo ha poteri limitati, che sarebbero due funzioni principali: l'esercizio di governo attraverso l'elezione del primo ministro e la promulgazione di leggi che devono essere adottate dal Parlamento. In legislativa il Consiglio Supremo approvava le leggi che si voteranno dai rappresentanti e adottava misure per la loro attuazione, mentre in relazione alla formazione del nuovo governo, in caso di dimissioni o licenziamento, il Consiglio Supremo nominava il primo ministro, che insieme con il governo formato da sé, dovrebbe prendere il consenso dei rappresentanti (che poteva rifiutarsi fino a tre volte il primo ministro).

I Delegati del Congresso di Lushnja, per il modo del funzionamento degli organi statali approvarono il documento costituzionale "Le basi del Canone di Consiglio Supremo", che stabilisce i principi costituzionali dello Stato, le caratteristiche di base della costituzione 0 dello statuto, da dove ha preso il nome con il quale si riconosce nella storia dello stato albanese "Statuto di Lushnja". Questo statuto, in quanto il documento costituzionale (in particolare con conseguente espansione), per il tempo e le condizioni dell'Albania, è stato un documento molto avanzato e democratico. Le sue disposizioni definiscono chiaramente il rafforzamento delle istituzioni del Capo dello Stato e delle sue relazioni con l'esecutivo e il Senato. ${ }^{5}$

Negli ultimi due punti, ovviamente, il potere dominante era il Senato, poi con la nomina del nuovo Consiglio Nazionale, cioè il primo parlamento albanese, indipendente dal Consiglio Supremo. Anche se non sono stati eletti i suoi 37 membri, loro sono stati membri adottati da rappresentativi di ogni provincia. II suo compito era l'attività legislativa dello stato albanese, attraverso l'approvazione di leggi che il Consiglio Supremo non può né annullare, né restituire al Consiglio Nazionale per la discussione. II suo compito successivo è stata la nomina e la revoca del governo. La formazione del nuovo governo, a seguito della nomina da parte del Consiglio Supremo, dovrebbe prendere il voto di fiducia del Consiglio Nazionale. Quando il voto di fiducia si rifiutava, allora si procedeva con la formazione di un altro governo, e quando si rifiutavano di fila due governi, poi si considerava come un conflitto sorto tra il Consiglio Supremo e il Consiglio Nazionale. ${ }^{6}$

Secondo il ricercatore Gramoz Hysi, se analizziamo la situazione giuridica dei tre organi statali più alti, il Consiglio Supremo, il Senato (Consiglio Nazionale) e il Governo, creati nel Congresso di Lushnja, si nota che "nella loro formazione è seguita il principio della separazione dei poteri"7.

In conclusione possiamo dire che il Congresso di Lushnja costituisce un evento di importanza storica perché ha gettato le basi per la costruzione della vita parlamentare, ha deciso le origini di uno stato organizzato in termini di separazione dei poteri, risultato questo degli sforzi delle forze politiche democratiche e patriottico albanese per impostare il nuovo stato albanese in basi costituzionali, secondo i principi riconosciuti degli stati liberali e democratici in Europa.

\section{I Lavori del Nuovo Parlamento Albanese}

Congresso di Lushnja ha gettato le basi del nuovo stato albanese e sarebbe un punto di partenza per lo sviluppo continuo delle attività degli organi statali. Da questo momento inizia il lavoro per organizzare tutti i rami amministrativi del governo che avrebbe diretto tutti i settori vitali della società albanese. Compito principale era quello di redigere la costituzione come un atto di base di stato che sarebbe in fatto l'espansione dello Statuto di Lushnja, anche l'elaborazione

qysh në fillim, si parlament i shtetit shqiptar. Për më tepër qysh në Lushnjë lindin dyshime për saktësinë e emrit të këtij organi, ndërsa më vonë gjithmonë e më shumë theksohet se "ky është parlament e jo senat". Me një cilësi të tillë, si "të parin parlament të shtetit shqiptar" e përshëndeste edhe Këshilli i Lartë fillimin e punimeve të Senatit të zgjedhur në Lushnjë. Në mbledhjen e parë të këtij organi u vendos që ai "të ketë trajtë parlamentare" dhe që "emri i tij të jetë Këshilli Kombëtar"." Grup autorësh, Historia e shtetit dhe e së drejtës në Shqipëri (Botimi III). Shtypshkronja universitare "Luarasi”, Tiranë 2005, pg.333.

${ }^{4}$ Afrim Krasniqi, Sistemet politike në Shqipëri 1912-2008. Shtëpia Botuese UFO Press, Tiranë, mars 2009, pg.68-70.

${ }^{5}$ Afrim Krasniqi, Sistemet politike në Shqipëri 1912-2008. Shtëpia Botuese UFO Press, Tiranë, mars 2009, pg.70-71.

${ }^{6}$ Grup autorësh, Historia e shtetit dhe e së drejtës në Shqipëri (Botimi III). Shtypshkronja universitare "Luarasi", Tiranë 2005, pg.334.

${ }^{7}$ Grup autorësh, Historia e shtetit dhe e së drejtës në Shqipëri (Botimi III). Shtypshkronja universitare "Luarasi", Tiranë 2005, pg.334. 
di una nuova legislazione, al posto del quello esistente ottomano. II Congresso di Lushnja, attraverso queste funzioni, ha iniziato il processo di europeizzazione istituzionale e legislativo dello stato albanese, già indipendente. In realtà, questi problemi vedremo che porteranno disagi all'interno delle forze politiche.

Dopo aver definito i nomi dei deputati, il 5 febbraio e impostato la prima riunione del nuovo parlamento, che ha iniziato a lavorare al suo primo incontro con il giuramento dei deputati, il 27 marzo 1920. Nella cerimonia di apertura hanno partecipato autorità civili, militari e tutte le persone. II Parlamento ha tenuto nella prima sessione dal 27 marzo al 27 maggio al 43 sessioni e la seconda sessione dal 23 settembre al 15 novembre al 27 sessioni.

Tra le prime questioni discusse in parlamento ci fu l'organizzazione interna del parlamento, la redazione del regolamento interno con 72 articoli e 13 capitoli 8 ; sono stati organizzati comitati per il lavoro amministrativo e militare, della finanza e post - telegrafo, l'istruzione, lavori pubblici e giustizia politica; si è deciso di pubblicare la gazzetta ufficiale per la pubblicazione delle leggi, le discussioni e le decisioni del parlamento; sono stati fissati norme per la tutela e la garanzia dell'immunità parlamentare, la tutela della sua libertà ed attività. ${ }^{9}$

In base alle norme stabilite dal Parlamento, le leggi del governo e le iniziative dei parlamentari entro cinque giorni dovrebbero essere esaminati in commissioni parlamentari e dovrebbero essere approvato dal 2/3 dei voti. La votazione è aperta con carta bianca (pro) e carta nera (contro), mentre il voto segreto era una cassa chiusa senza i nomi. ${ }^{10}$

Questo Parlamento ha esercitato un potente controllo parlamentare sul governo. I deputati hanno cercato di ottenere più autorità e potere attraverso la pressione parlamentare sul governo, che aveva potere esecutivo e amministrativo. Particolare importanza, soprattutto in termini di creazione di tradizione della separazione dei poteri, avevano i rapporti del parlamento con il Consiglio Supremo. Quest'ultimo inviava per ogni apertura della sessione parlamentare un messaggio di benvenuto. A questo messaggio il parlamento ha risposto per iscritto dove ha cercato da parte del Consiglio Supremo di esercitare pressioni sul governo per intervenire sulla situazione nel paese, una pratica questa che oggi non è implementata in Albania, ma ha continuato ad essere applicata in alcune democrazie europee. Nel corso del tempo i dibattiti politici interni che hanno luogo nel governo e il Consiglio Supremo si sono sentiti anche in parlamento. Al suo interno è venuto un gruppo di opposizione che non è d'accordo con il governo. Qui hanno avuto origine due nuovi partiti politici in Albania: il Partito Popolare e il Partito Progressista. ${ }^{11}$

Quindi, anche se in forma embrionale, e tra le molte difficoltà, a causa della sottolineata arretratezza del passato, sono chiaramente visibili le caratteristiche fondamentali di un governo del tipo parlamentare. Questo Parlamento è in qualche modo espressione di un governo rappresentativo anche se costituito da elementi non eletti direttamente dal popolo, ma dai suoi rappresentanti. II Consiglio Nazionale è stato un potere legislativo come progettare e approvare leggi tramite la votazione della maggioranza. Tra potere legislativo ed esecutivo c'erano rapporti di fiducia e di recente al suo interno sono stati creati dei partiti politici che avranno la loro influenza sui successivi sviluppi. Per quanto riguarda il ruolo degli elettori, si sentirà nelle fasi successive.

In conclusione possiamo dire che, anche se ha avuto le sue caratteristiche distintive, il tipo di governo parlamentare che si stava stabilendo in Albania portava per sé le caratteristiche fondamentali comuni che definiscono un tale tipo di governo.

Nel 17 dicembre del 1920, l'Albania è stata ammessa alla Società delle Nazioni, così si e ottenuto il riconoscimento internazionale. Già, secondo la decisione del Congresso di Lushnja, si richiedeva la selezione di una Assemblea Costituente per determinare la forma di governo. Questa decisione ha provocato dibattito in parlamento, poiché parte dei parlamentari ha chiesto nuove elezioni perché in questo momento dovrebbe essere presa la fiducia dei cittadini attraverso libere elezioni. Secondo le voci più avanzate doveva essere rispettata lo Statuto di Lushnja, che prevedeva un'Assemblea Costituente eletta al posto del Consiglio Nazionale, che avrà le funzioni complete del parlamento e avrebbe una base più ampia rispetto al Consiglio Nazionale eletto al momento del Congresso di Lushnja. I dibattiti in parlamento avevano due linee opposte di atteggiamento in congiunzione con chi avrebbe redatto la legge elettorale, il governo o una commissione di membri del parlamento. Ha vinto la linea del comitato dei deputati, che sarebbe composto da quattro membri, due deputati e due membri di governo. Nel 14 novembre è stato fissato per sciogliere il parlamento e convocazione delle elezioni, ma il giorno dopo le dimissioni del governo Delvina, il governo si ritirò perdendo così la maggioranza in parlamento. Nello stesso giorno il nuovo parlamento sceglierà un nuovo Primo

\footnotetext{
8 “...Ndonëse ai ishte një akt që duhej të rregullonte veprimtarinë e parlamentit, ai i kapërcente kufijtë dhe vendosi një parim kushtetues, duke sanksionuar se Këshilli Kombëtar me 2/3 e anëtarëve të tij mund të përpunonte zgjerimin e Statutit të Lushnjës." Arben Puto, Shqipëria politike 1912-1939. Botimet Toena, Tiranë, 2009, pg.315.

${ }^{9}$ Afrim Krasniqi, Sistemet politike në Shqipëri 1912-2008. Shtëpia Botuese UFO Press, Tiranë, mars 2009, pg.73.

${ }^{10}$ Afrim Krasniqi, Sistemet politike në Shqipëri 1912-2008. Shtëpia Botuese UFO Press, Tiranë, mars 2009, pg.73.

${ }^{11}$ Afrim Krasniqi, Sistemet politike në Shqipëri 1912-2008. Shtëpia Botuese UFO Press, Tiranë, mars 2009, pg.74.
} 
Ministro, lliaz Vrioni con mandato temporaneo fino all'elezione del nuovo parlamento che eletto il nuovo governo. Dopo la votazione del governo Vrioni, praticamente il parlamento non è più stato convocato ed era il governo che ha amministrato la vita politica del paese.

II Ministero degli Interni, senza chiedere il parlamento e contrario ai principi costituzionali di separazione dei poteri, nel 2 dicembre ha annunciato per sciogliere il parlamento. II 5 dicembre 1920 si e approvata la legge "Sulla elezioni dei deputati ", sulla base dei quali si sono tenute le elezioni per il Consiglio Nazionale, le prime del suo genere in Albania, che saranno reso con voto indirettamente, attraverso un sistema elettorale misto e elezioni in due fasi. Per quanto riguarda il diritto di voto, la legge in questione aveva abbastanza restrizioni e non eccelleva dei principi democratici. II diritto di voto l'avevano solo i maschi di età superiore ai 20 anni che avevano vissuto per più di 6 mesi nella zona di voto. ${ }^{12}$ La legge escludeva le donne dal voto e l'esercito e aveva le restrizioni basato sul reddito da patrimonio. Una limitazione importante per le condizioni che hanno caratterizzato la società albanese di questo periodo è stata la analfabetismo della maggioranza della popolazione, perché secondo la legge, perché nelle schede elettorali erano stati scritti i nomi dei candidati e dei partiti, il nome scelto dovrebbe essere scritto con la mano da l'elettore 0 da una terza persona. Di ogni 500 elettori veniva fuori un secondo elettore con età da oltre 25 anni, e da i secondi elettori, per ogni 12 mila persone si sceglieva un elette più di $30 \mathrm{anni}^{13}$ (anche se questo criterio non è stato applicato in generale, la prova di questo è l'elezione di Ahmet Zogu all'età di 26). Dai gruppi politici concorrenti si è cercato di tener conto anche dei criteri religiosi come indicatori per l'armonia interreligiosa nel paese. Tutte queste restrizioni influenzeranno la composizione del Parlamento, dove la maggioranza dei parlamentari erano i rappresentanti degli interessi di classe di bey.

Le elezioni si sono svolte nei mesi di febbraio-marzo 1921 e sono concluse nel 5 aprile 1921. Queste sono le prime elezioni democratiche e multipartitiche nella storia dell'Albania, dove per la prima volta hanno partecipato dei diversi gruppi politici, dove le più grande erano: il Partito Progressista, il Partito Popolare e un gruppo di parlamentari indipendenti che hanno fatto alleanza con il Partito Popolare. II Parlamento aveva 78 membri, di cui 77 sono stati deputati eletti e un deputato era nominato dalla società "Vatra" negli Stati Uniti. II Partito Popolare ha ottenuto 39 seggi, il Partito Progressista 30, mentre gli indipendenti 8 seggi. Con la creazione di questi partiti e la loro rappresentanza in parlamento in Albania per la prima volta si è creato un sistema bipartitico a malapena abbastanza distinguibili.

Gli anni 1921-1924 furono anni di crisi politica e il governo ${ }^{14}$ è stato caratterizzato dallo spirito di scisma in gruppi all'interno dei partiti, dalle irritazione dei rapporti occasionali tra il governo, il parlamento e il Consiglio Supremo, dalle contraddizioni tra le forze politiche, soprattutto in connessione con il controllo parlamentare sul governo e il Consiglio Supremo, e in connessione con l'elaborazione di una nuova legislazione. Venuta meno agli obblighi del codice civile che era molto necessario per la regolamentazione dei rapporti di proprietà in momenti del attraversamento di rapporti feudali. I risultati di questo periodo sono collegati con i settori del diritto finanziario, amministrativi e penali, organizzazione della giustizia in tribunale di tre gradi, pubblicazioni dei minuti di dibattiti parlamentari e con la proposta di parlamentari progressisti nei primi mesi del 1921, è stato istituito un Comitato ad hoc di 12 persone per l'adempimento dello Statuto di Lushnja.

Nel 14 dicembre 1922 è stato approvato un documento costituzionale in forma completa e con la gamma espansa che ha preso il nome di "Statuto Espanso di Lushnja", che sarà provvisoriamente in vigore fino all'approvazione dello Statuto dello stato da parte dell'Assemblea Costituente. In questo documento è stato determinato la forma monarchica di governo; è stato sanzionato il principio della sovranità popolare; sono stati definiti i poteri degli organi più alti statali; sono stati definiti compiti e poteri del parlamento, i suoi rapporti con il Consiglio Supremo e il governo; sono stati generalmente trattati diritti elettorali; è stato determinato il posizionamento costituzionale e giuridico del Consiglio Supremo, del governo come un organismo esecutivo; è stato sanzionato l' indipendenza del tribunale, sono state affrontate le questioni dei diritti

\footnotetext{
12 Valentina Duka, Histori e Shqipërisë 1912-2000. Shtëpia Botuese e Librit Universitar, Tiranë, 2007, pg.129.

13 "Shprehur në shifra, deputeti duhej të siguronte shumicën e votave të 24 zgjedhësve të dytë, duke u bërë njëherësh përfaqësues i 12 mijë banorëve. Kështu, çdo delegat i dytë përfaqësonte 500 banorë, dhe çdo deputet 24 delegatë të dytë". Afrim Krasniqi, Sistemet politike në Shqipëri 1912-2008. Shtëpia Botuese UFO Press, Tiranë, mars 2009, pg.78.

14 "Nga nëntori i vitit 1920 dhe shkurti i vitit 1922, pra, brenda 16 muajve u krijuan 8 qeveri shqiptare. Vetëm më 1921-in u krijuan gjashtë të tilla, madje në dhjetor 1921 u krijuan katër kabinete qeveritare. Në dy raste kryeministra ishin lljaz Vrioni dhe në një rast Hasan Prishtina, Pandeli Vangjeli, Idhomenë Kosturi, Qazim Koculi, Xhafer Ypi e Ahmet Zogu....Rekordin e qeverisjes e mban përsëri Ahmet Zogu, I cili drejtoi qeverinë midis shkurtit 1922 dhe marsit 1924, pra rreth dy vjet, dy here më shumë se Vrioni dhe 1.5 herë më shumë se shtatë kryeministrat e tjerë të marrë së bashku. Afrim Krasniqi, Sistemet politike në Shqipëri 1912-2008. Shtëpia Botuese UFO Press, Tiranë, mars 2009, pg.83."
} 
e delle libertà dei cittadini , ecc. . $^{15}$

\section{II Processo Elettorale del 1923}

Sulla base dello " Statuto Espanso di Lushnja " il processo elettorale si svolgerà una volta ogni quattro anni, ma escludendo le prossime elezioni (le quali dalle decisioni del Congresso di Lushnja si terranno dopo un periodo di due anni). Quindi, in questa clima così appesantito dalle crisi di governo, nel settembre del 1923, il governo di Ahmet Zogu ha elaborato la proposta di legge per le elezioni dell'Assemblea Costituente, "Per la scelta dei delegati dell'Assemblea Costituente". Dopo molte discussioni feroci tra i sostenitori del governo e l'opposizione, dopo le dichiarazioni di quest'ultimo di boicottare le elezioni e dopo ripetuti casi di boicottaggio di sessioni parlamentari a sostegno della loro richiesta di dimissioni del governo ${ }^{16}$, la legge esaminata dalle commissioni parlamentari di giustizia e l'amministrazione, è venuto per essere adottato nel 30 settembre 1923.

Nella nuova legge con 94 articoli non c'è stato alcun cambiamento da quella del 1921. Era salvato il sistema elettorale di due gradi, dove gli elettori sceglieranno la seconda elettore, i quali avrebbero votato la lista dei candidati per i deputati. Si è diminuita l'età degli elettori (uomini, perché le donne, così come i poveri, i disabili, detenuti, militare ecc., non erano del diritto di voto.) da 20 anni a 18 anni e si potevano essere selezionati coloro che avevano raggiunto l'età di 25 anni; come conseguenza di aumentare il numero dei parlamentari da 78-102, per ogni 800.000 residenti avrebbe un deputato, mentre per il secondo elettore votavano 250 abitanti. II primo voto sarebbe durato 25 giorni e sarebbe diventato con le sfere 0 granelli come conseguenza dell'alto tasso di analfabetismo nel paese, mentre l'elezione dei deputati sarebbe lo stesso giorno e con la scheda elettorale. ${ }^{17}$

Le modifiche erano apportate anche nell'organizzazione e nella gestione del processo elettorale. Cinque giorni dopo il giudizio sulla data delle elezioni, in tutti i centri amministrativi sarebbe stabilito un comitato di tre membri: un tribunale provinciale, un impiegato comunale e un funzionario pubblico, che dichiareranno e prepareranno le liste degli elettori. Le liste saranno verificati da ciascun partito, candidati e cittadini. Un comitato elettorale di tre membri, un giudice locale e da due membri eletti dal consiglio comunale, impegnerebbero la gestione dei voti. L'annuncio ufficiale dei risultati delle votazioni sarebbe la decisione del Ministero degli Interni. La legge contiene anche modifiche ricorsi contro le irregolarità e la condanna dei violatori della legge elettorale. ${ }^{18}$

Le forze politiche che hanno partecipato alle elezioni del 1923 hanno mantenuto le posizioni di una politica ambigua e continuavano ad agire più sulla base degli interessi di particolari persone, e non sulla base di un programma politico definito. La maggior parte di loro sono recati alle urne nemmeno con un programma elettorale, ma la loro campagna è stata basata solo su promesse. Queste forze, le cosiddette "partito", sono stati collegati in gruppi, che il più grande era "il Braccio Nazionale", o altrimenti conosciuto come "Cricca" e in seguito "Gruppo Governo", basato sul Partito Popolare. ${ }^{19}$

La campagna elettorale si è conclusa il 27 dicembre 1923, con l'elezione di 102 deputati. Si è svolto in condizioni difficili, ci sono stati diversi incidenti, violenza e abbastanza manipolazione. Il lato governativo (sostenitori di Zog) ha ottenuto la maggioranza, ma non un numero sufficiente di parlamentari per formare il governo. L'Assemblea Nazionale (il parlamento) ha iniziato ad operare in 21 gennaio 1924. Durante il breve periodo del suo funzionamento, da gennaio a giugno 1924 si è riunita 63 volte. II primo compito dell'Assemblea Nazionale, stabilito nel Congresso di Lushnja, è stata la decisione sulla forma di governo in Albania. Sebbene questa questione è stata discussa in parlamento, è stato istituito anche un gruppo parlamentario di 28 deputati per preparare un progetto. Come risultato di opinioni e atteggiamenti contrastanti, sono stati presentati due progetti. Per decidere quale di loro sarebbe più conveniente per il paese, più determinante sarebbe consenso sulla questione della forma di governo, invece di definire la decisione di voto. Un'altra causa di irritazione tra le parti è stato il rifiuto di Ahmet Zogu di dimettersi, violando così lo "Statuto Espanso di Lushnja", che prevedeva che dopo le prossime elezioni, il governo e il primo ministro dovrebbero votare in parlamento.

La situazione politica in Albania era molto irritata e piena di tensioni politiche tra i sostenitori di Zog e l'opposizione. In una tale situazione di tensione e incidenti, la vita parlamentare sarebbe finita con la dissoluzione del parlamento, il 10

\footnotetext{
15 Per di piu vedi anche: -Arben Puto, Shqipëria politike 1912-1939.Botimet Toena, Tiranë, 2009, pg.318-320. -Grup autorësh, Historia e shtetit dhe e së drejtës në Shqipëri (Botimi III). Shtypshkronja universitare "Luarasi”, Tiranë 2005, pg.335-343. -Afrim Krasniqi, Sistemet politike në Shqipëri 1912-2008. Shtëpia Botuese UFO Press, Tiranë, mars 2009, pg.87-89.

${ }^{16}$ Aurela Anastasi, Institucionet politike dhe e drejta kushtetuese në Shqipëri. Shtëpia Botuese "Luarasi", Tiranë, 1998, pg.139.

${ }^{17}$ Afrim Krasniqi, Sistemet politike në Shqipëri 1912-2008. Shtëpia Botuese UFO Press, Tiranë, mars 2009, pg.92-93. Per di piu vedi anche: Aurela Anastasi, Institucionet politike dhe e drejta kushtetuese në Shqipëri. Shtëpia Botuese "Luarasi", Tiranë, 1998, pg.145.

${ }^{18}$ Afrim Krasniqi, Sistemet politike në Shqipëri 1912-2008. Shtëpia Botuese UFO Press, Tiranë, mars 2009, pg.92-93.

19 Valentina Duka, Histori e Shqipërisë 1912-2000. Shtëpia Botuese e Librit Universitar, Tiranë, 2007, pg.142.
} 
giugno 1924. Durante il governo di Fan Noli20, che è venuto a capo del governo dopo la rivoluzione di giugno, non c'era vita parlamentare. II Parlamento non è mai stato convocato. II governo ha promesso che dopo la pacificazione della situazione ci sarebbe stata una nuova elezione parlamentare a scrutinio segreto e diretto, ma la sua breve vita non ha reso possibile la realizzazione di questa promessa.

\section{Bibliografia}

Afrim Krasniqi, Sistemet politike në Shqipëri 1912-2008. Shtëpia Botuese UFO Press, Tiranë, mars 2009.

Arben Puto, Shqipëria politike 1912-1939. Botimet Toena, Tiranë, 2009.

Aurela Anastasi, Institucionet politike dhe e drejta kushtetuese në Shqipëri. Shtëpia Botuese "Luarasi", Tiranë, 1998.

Grup autorësh, Historia e shtetit dhe e së drejtës në Shqipëri (Botimi III). Shtypshkronja universitare "Luarasi", Tiranë 2005.

Nasho Jorgaqi, Jeta dhe vepra e Fan. S. Nolit (vëllimi I, II). Tiranë, 2005.

Romeo Gurakuqi, Shqipëria dhe çështja shqiptare pas Luftës së Parë Boterore. Botimet "Camaj-Pipa", Shkodër, 2007.

Valentina Duka, Histori e Shqipërisë 1912-2000. Shtëpia Botuese e Librit Universitar, Tiranë, 2007.

${ }^{20}$ Di piu per la figura di Fan Noli vedi anche: Nasho Jorgaqi, Jeta dhe vepra e Fan. S. Nolit (vëllimi I, II). Tiranë, 2005. 